\title{
COMPARISON OF DIFFERENT SURGICAL PROTOCOLS IN MANAGEMENT OF MEDICATION-RELATED OSTEONECROSIS OF THE JAW USING PLATELET-RICH FIBRIN AND BONE MORPHOGENETIC PROTEIN-2
}

\author{
Hesham Fattouh* and Mohamed Ashraf*
}

\begin{abstract}
Objectives: The aim of this study is to compare the effect of platelet-rich fibrin (L-PRF) and bone morphogenetic protein-2 (rhBMP-2) in management of medication-related osteonecrosis of jaw (MRONJ).

Patients and Methods: Among 35 patients who were diagnosed with MRONJ, 12 patients were treated with L-PRF, 12 patients were treated with rhBMP-2 and 11 patients were treated with both L-PRF and rhBMP-2, evaluation of surgical sites was done for each patient postoperatively at 4 and 16 weeks.
\end{abstract}

Results: At 4 and 16 weeks postoperatively, MRONJ treated with both L-PRF and rhBMP-2 showed favorable outcomes with complete resolution of the lesions than that treated with L-PRF or rhBMP-2 alone. However this difference didn't reach the level of statistical significance $(\mathrm{P}=0.42)$.

Conclusion: The simultaneous use of both L-PRF and rhBMP-2 in management of MRONJ is a better treatment modality compared to the single use of each one of them alone.

KEYWORDS: Osteonecrosis of the jaw, PRF, rhBMP-2.

\section{INTRODUCTION}

Antiresorptive medicines, such as bisphosphonates (BPs), are broadly utilized in treatment of various bone diseases ranging from osteoporosis to metastatic bone disease ${ }^{(1)}$. The beneficial impacts of BPs include significant decreases in pain, pathologic fractures, osteolytic lesion size and the need for later bone surgery ${ }^{(2)}$. However, the long-term use of antiresorptive therapy is known to be associated with serious complication of medication-related osteonecrosis of the jaws (MRONJ) ${ }^{(3)}$. MRONJ is a potentially genuine condition characterized by exposed necrotic bone in the mandible or maxilla for more than 8 weeks in a patient who has taken or is right now taking therapeutic BPs and who has no history of therapeutic radiation to the jaws, it is

\footnotetext{
* Lecturer of Oral and Maxillofacial Surgery, Faculty of Oral and Dental Medicine, Cairo University.
} 
usually associated with pain, swelling, paresthesia, suppuration and soft tissue ulceration ${ }^{(4-5)}$. The incidence of MRONJ is greater with BPs administered through a parenteral route than with oral BPs ${ }^{(6-7)}$.

Ideally, we should avoid any traumatic dental procedures within the bone after begin of the BPs treatment as prevention is the most important aspect of this condition. However, if MRONJ occurs, various methods of treatment are available depending on the degree of bone exposure and spread to adjacent structures. Nevertheless, there is currently no gold standard for the treatment of MRONJ ${ }^{(8)}$, conservative approaches including antimicrobials mouthwashes and antibiotics are reasonable line of treatment to control pain and infection $^{(9-10)}$. If surgical treatment is indicated, effective surgery should improve the MRONJ prognosis $^{(11)}$, the extent of necessary surgery has shown a lot of debate, despite debates regarding the surgical treatment of MRONJ, several authors have reported surgical cure vary from 59\% to more than $90 \%$, which have encouraged doctors to continue dealing with MRONJ surgically. However, the data on the surgical management of MRONJ are very limited and usually associated with a single center $^{(12-13)}$.

Platelet-rich fibrin (PRF) is a biotechnology that is utilized for the stimulation and acceleration of the process of tissue healing and bone regeneration that was first described by Choukroun et al in $2000^{(14)}$. It regulates inflammation process and also stimulates chemotactic factors involved in body immune response, PRF also accelerates angiogenesis and the multiplication of both fibroblasts and osteoblasts ${ }^{(15)}$. In recent years, PRF has been used as an autologous grafting material due to its ability to accelerate both physiologic wound healing and new bone formation processes; it acts as a barrier membrane between the alveolar bone and the oral cavity. PRF offer a quick, simple and successful alternative method for bone exposure closure in MRONJ ${ }^{(16)}$.
Bone morphogenetic protein (BMP) was depicted first by Urist and Strates ${ }^{(17)}$ as a substance that is extracted from bovine bone and have the ability to induce bone formation in rat muscle pouches $^{(18)}$. A recombinant human form of BMP-2 (rhBMP-2) loaded onto an absorbable collagen sponge is presently accessible and has been approved by the FDA for various surgical use $^{(19)}$. BMP-2 stimulate osteoblast formation and activity during bone healing, also it increases bone remodeling ${ }^{(20)}$. Recently, there have been studies on the use of BMP-2 for the treatment of MRONJ to increase bone remodeling process ${ }^{(21)}$. Consequently BMP-2 as a bone remodeling enhancement factor, is thought to have an incredible inversion impact on the remodeling-suppressed bone in MRONJ ${ }^{(22)}$.

There is a lack of research on the simultaneous use of BMP-2 and L-PRF with single use of L-PRF or the single use of BMP-2 in the healing treatment outcome of MRONJ which we tried to emphasize in this research.

\section{PATIENTS AND METHODS}

\section{Study design and data collection}

In our study we followed the guidelines of the World Medical Association Declaration of Helsinki 2000 for biomedical research involving human subjects. The nature of the performed procedure with its risks, benefits and possible complications were explained to the patients and a written consent was obtained from the patient for their approval of doing the operation.

A prospective study was performed on 35 patients (9 males, 26 females) with ages ranged from 46 to 68 years with a mean of age of 59 years. Patients were selected and categorized randomly from the outpatient clinic of the Department of Oral and Maxillofacial Surgery, Faculty of Oral and Dental Medicine, Cairo University. The inclusion criteria were: patients who were, or who are on current treatment with bisphosphonates and were diagnosed with MRONJ with sites that had failed 
to heal for 8 weeks or longer despite continuous treatment with antimicrobial drugs. The diagnosis was confirmed clinically: by the presence of necrotic bone in the jaw which is needed to be surgically removed (Fig. 1A), and radiographically using panorama and computed tomography (CT): by the presence of destructive bony lesion with sequestra (Fig. 1B), radiographs were used also to assess lesions extensions. Patients with history of radiation therapy to the jaws or who had neoplastic involvement of the jaw were excluded.

Thorough medical and dental history taking, risk factors, coexisting medical conditions including patient-related (diabetes, obesity and renal failure) and iatrogenic factors (use of steroids and chemotherapy) were documented. Type, dose, duration, route of administration and reason for bisphosphonates therapy were recorded. The site and size of the exposed necrotic bone, the presence of infection and pain and the extension of lesions were also recorded.

\section{Staging of the lesions were performed according to American Association of Oral and Maxillofa- cial Surgeons (AAOMS) clinical classification of MRONJ $^{(3)}$ :}

Stage 1: exposed and necrotic bone or fistula that probes to bone in patients who are asymptomatic and have no evidence of infection.
Stage 2: exposed and necrotic bone or fistulas that probes to bone associated with infection as evidenced by pain and erythema in the region of exposed bone with or without purulent drainage.

Stage 3: exposed and necrotic bone or a fistula that probes to bone extending beyond the region of alveolar bone in patients with pain, infection and one or more of the following: pathologic fracture, extraoral fistula, oro-antral or oro-nasal communication, or osteolysis extending to inferior border of the mandible or sinus floor.

\section{Surgical procedure:}

Our surgical protocol was to remove all infected tissue of both necrotic sequestrum and granulation tissue first by using surgical curettes until enhancing fresh bleeding from bone, later by rotary instrument for osteoctomy and rounding any sharp bony edges, this was followed by intensive irrigation of broad spectrum-antibiotic solutions ( $2 \mathrm{~g}$ of third-generation cephalosporin in $1 \mathrm{~L}$ saline), aiming to minimize remaining bacterial contamination and remove of any debris or foreign bodies. Patients were randomly assigned into three groups according to intended material that would be placed on the bony defect. Group A were treated with L-PRF only, Group B were treated with rhBMP-2 on a collagen sponge carrier, while Group C were treated by both rhBMP-2 and PRP.

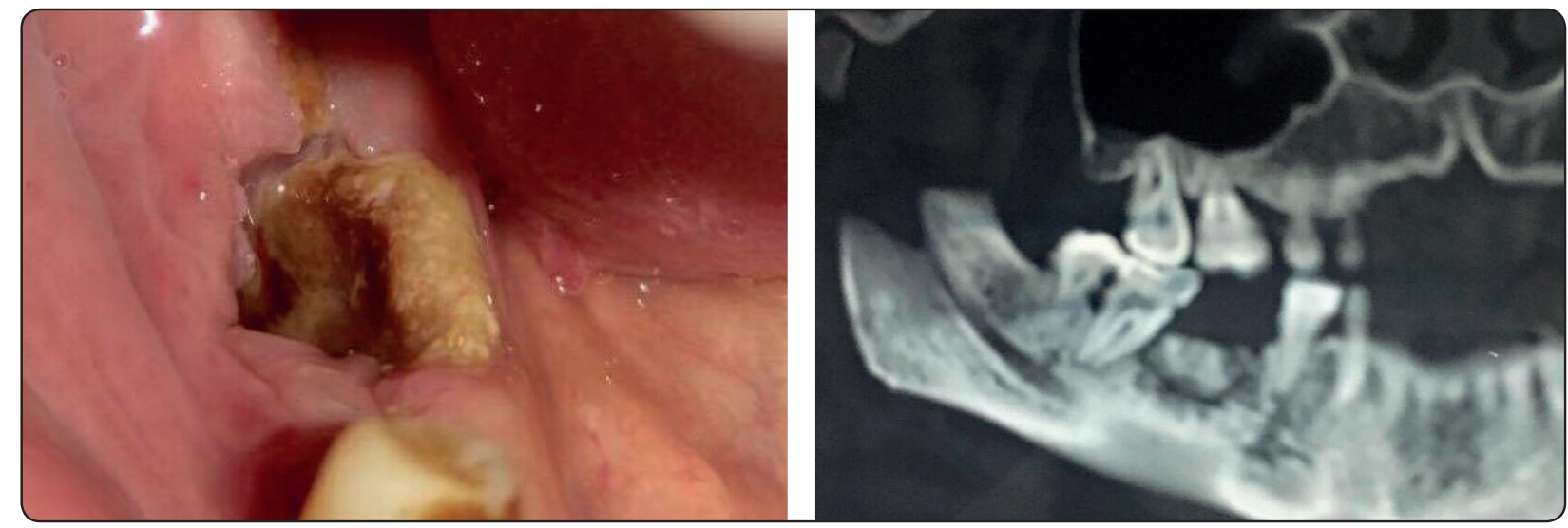

Fig. (1) (A) Preoperative photograph shows a lesion of MRONJ. (B) Preoperative radiograph shows a lesion of MRONJ. 
In Group A: The PRF protocol was performed according to the Choukroun procedure and European Directive 2004/ 23/CE of March 31, 2004. To prepare the L-PRF, we collected $20 \mathrm{ml}$ of the patient own venous blood. A blood sample was then divided in two $10-\mathrm{mL}$ glass test tubes with no anticoagulant, and was immediately centrifuged at $3000 \mathrm{rpm}$ for 10 minutes to prevent initiation of the coagulation cascades before centrifugation. After centrifugation, L-PRF was obtained from the middle of the tube (Fig. 2A), exposed bone was covered with 2 layers of PRF membrane (the first layer of the PRF membrane was inserted into the alveolar bone cavity while the second layer was placed superficially under the mucoperiosteal flap and sutured carefully to the surrounding gingiva with 4.0 Vicryl sutures for stabilization) (Fig. 2B)

In Group B, (rhBMP-2) loaded onto an absorbable collagen sponge carrier*(1/2 inch $\mathrm{x} 1$ inch $x 7.0 \mathrm{~mm}$ ) was inserted in direct contact with the exposed bony surface.

Group C patients received both rhBMP-2 and L-PRF simultaneously, first collagen sponge sections with rhBMP-2 were placed in direct contact to the bone surface, then L-PRF application was done. Primary closure of the mucoperiosteal was done in all groups.

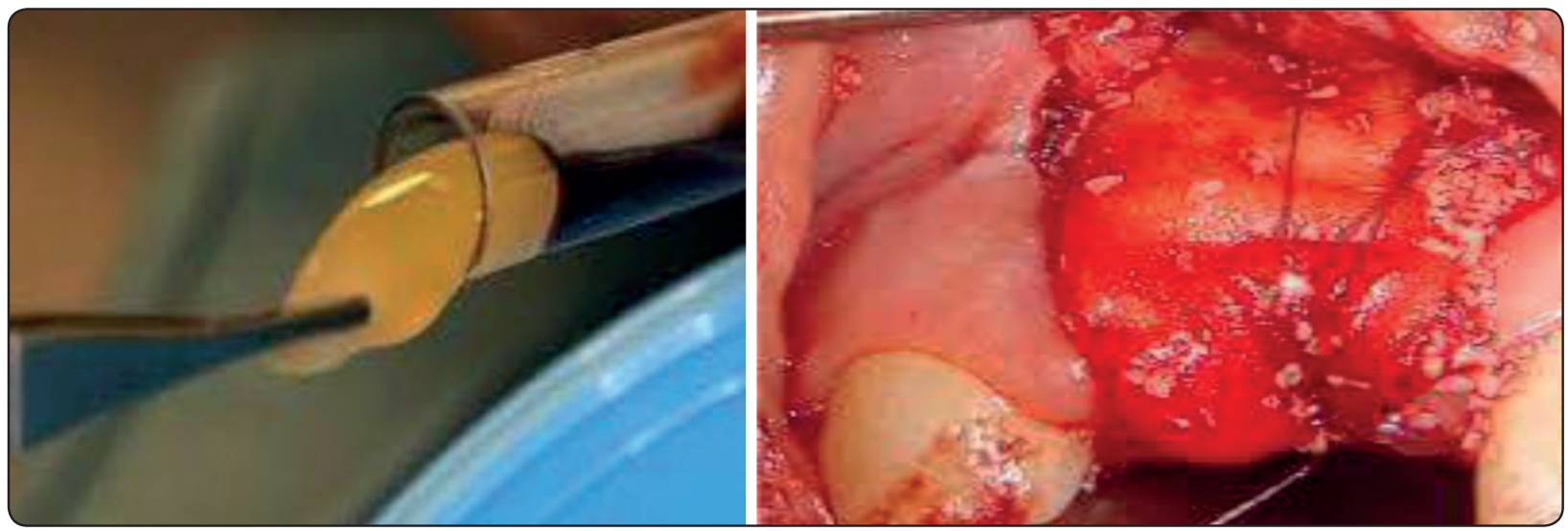

Fig. (2) (A) L-PR obtained by collecting blood in a tube free of anticoagulant and immediately centrifuging. (B) L-PR put in the bony defect.

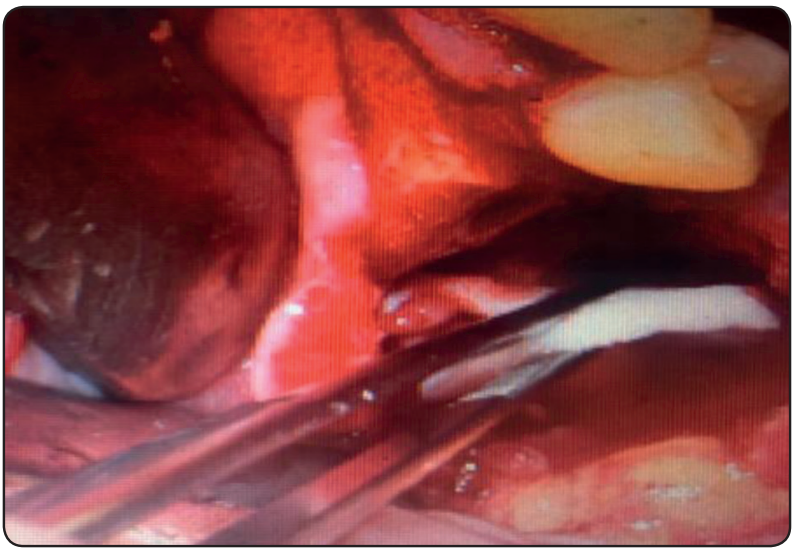

Fig. (3) Collagen sponge pieces with rhBMP-2 inserted with direct contact to bone surface.
All patients used antibacterial mouth wash till complete healing and systemic antibiotics for 3 weeks postoperatively. Patients were given standard postoperative instructions and instructed not to brush the teeth in the treated area but to gently clean the wound using gauze impregnated with $3 \%$ hydrogen peroxide 3 times daily for 2 weeks. A cold semi liquid diet for the first day was suggested. Normal oral hygiene procedures were re-established after 3 days.

* Helistat absorbable collagen $(1 / 2$ inch x 1 inch x $7.0 \mathrm{~mm})$ manufactured by Integra life sciences corporatin (FDA approved). 


\section{Assessment of response to treatment}

To confirm secondary healing of the surgical sites, follow up visits were scheduled weekly for the first month then monthly for 6 months. At the 16 weeks follow-up, a new dental panoramic radiograph and a CT scan were obtained. Complete resolution was defined as no exposed or necrotic bone at the site, full coverage by mucosa and no pain at 1 month. Resolution was "delayed" when exposed or necrotic bone were present at 1 month but had resolved completely by 16 weeks. No resolution was defined as persistence of symptoms at the 16 weeks follow-up.

\section{Statistical analysis}

Statistical analyses were performed using SPSS, Version 16.0 (SPSS Inc., Chicago, IL, USA). Age and duration of treatment were expressed as mean and standard deviation. These data were normally distributed (parametric data), therefore one way analysis of variance (ANOVA) test was used to compare between groups. Values of all other parameters were presented as number and percentage and Chi square test was used for comparison between groups. All values were considered statistically significant when $P<0.05$.

\section{RESULTS}

The study included 35 patients (12 in group A, 12 in group B and 11 in group C. Baseline characteristics between the three groups are shown in Table 1. The patients were well matched between the groups regarding age, sex, observation period, reason, type, duration and route of administration for antiresorptive agents, dental etiologic factors and other risk factors for MRONJ development, AAOMS stages and site of MRONJ. Age of all patients ranged from 46 - 68yrs, females constituted $66.7 \%, 75 \%, 82 \%$ of patients in the groups A, B, C respectively. Regarding the reason of antiresorptive therapy, osteoporosis was the main cause for all groups and constituted $58 \%, 75 \%, 64 \%$ of reasons in the groups $\mathrm{A}, \mathrm{B}, \mathrm{C}$ respectively.

The treatment outcomes are displayed in Table 2, the best outcome was noted in group C, where $73 \%$ of the patients showing complete resolution and only $9 \%$ showing no resolution. On the other hand, only $33 \%$ and $50 \%$ of patients showed complete resolution in groups A, B respectively. However this difference didn't reach the level of statistical significance $(\mathrm{P}=0.42)$

TABLE (1) Characteristics of study patients and significance of the difference

\begin{tabular}{|c|c|c|c|c|c|}
\hline & A:prf & B:bmp2 & C: ВОTH & P value & Test \\
\hline No of patients & 12 & 12 & 11 & ----- & \\
\hline Age (range) & $\begin{array}{c}58.2 \pm 5.39 \\
(46-66)\end{array}$ & $\begin{array}{c}58.5 \pm 7.81 \\
(46-68)\end{array}$ & $\begin{array}{c}55.5 \pm 6.8 \\
(48-67)\end{array}$ & 0.916 ns & ANOVA \\
\hline Sex & $\begin{array}{c}8 \text { female }(66.7 \%) \\
4 \text { male }(33.3 \%)\end{array}$ & $\begin{array}{c}9 \text { female }(75 \%) \\
3 \text { male }(25 \%)\end{array}$ & $\begin{array}{c}9 \text { female }(82 \%) \\
2 \text { male }(18 \%)\end{array}$ & $0.71^{\text {ns }}$ & Chi square \\
\hline Reason for antiresorptive therapy & $\begin{array}{c}\text { Osteoporosis } 7 \\
\text { Bone }(58 \%) \\
\text { metastasis } 5 \\
\mathbf{( 4 2 \% )}\end{array}$ & $\begin{array}{c}\text { Osteoporosis } 9 \\
\text { (75\%) } \\
\text { Bone metastasis } \mathbf{3} \\
\mathbf{( 2 5 \% )}\end{array}$ & $\begin{array}{c}\text { Osteoporosis } 7 \\
\text { (64\%) } \\
\text { Bone metastasis } 4 \\
\text { (36\%) }\end{array}$ & $0.68^{\mathrm{ns}}$ & Chi square \\
\hline
\end{tabular}




\begin{tabular}{|c|c|c|c|c|c|}
\hline & A:prf & B:bmp2 & C: ВОТН & $P$ value & Test \\
\hline $\begin{array}{l}\text { Duration of antiresorptive therapy } \\
\text { Range month }\end{array}$ & $\begin{array}{c}35.4 \pm 6.7 \text { months } \\
24-45\end{array}$ & $\begin{array}{c}39.7 \pm 9.3 \text { months } \\
24-50\end{array}$ & $\begin{array}{c}43 \pm 10.1 \text { months } \\
24-53\end{array}$ & $0.12^{\mathrm{ns}}$ & ANOVA \\
\hline $\begin{array}{l}\text { Route of antiresorptive agents } \\
\text { administration }\end{array}$ & $\begin{array}{c}\text { Orally:2 (16.7\%) } \\
\text { Intravenous:10(83\%) }\end{array}$ & $\begin{array}{c}\text { Orally } \mathbf{2}(\mathbf{1 6 . 7 \% )} \\
\text { Intravenous } \\
\mathbf{1 0}(\mathbf{8 3 \%})\end{array}$ & $\begin{array}{c}\text { Orally } \mathbf{3}(\mathbf{2 7 \%}) \\
\text { Intravenous } \mathbf{8}(\mathbf{7 3} \%)\end{array}$ & $0.77^{\mathrm{ns}}$ & Chi square \\
\hline \begin{tabular}{ll}
\multicolumn{2}{l}{ Type of antiresorptive therapy } \\
- & Zoledronate \\
- & Alendronate \\
- & Multiple
\end{tabular} & $\begin{array}{c}2(16.7 \%) \\
9(75 \%) \\
1(8.3 \%)\end{array}$ & $\begin{array}{c}4(33.3 \%) \\
8(66.6 \%) \\
0\end{array}$ & $\begin{array}{c}2(18 \%) \\
8(73 \%) \\
1(9 \%)\end{array}$ & $0.74^{\mathrm{ns}}$ & Chi square \\
\hline $\begin{array}{ll}\text { Dental etiologic risk factors } \\
\text { - } & \text { Surgical procedure } \\
\text { - } & \text { Ill fitting denture } \\
\text { - } & \text { Spontaneous }\end{array}$ & $\begin{array}{l}8(66.7 \%) \\
2(16.7 \%) \\
2(16.7 \%)\end{array}$ & $\begin{array}{l}6(50 \%) \\
5(42 \%) \\
1(8 \%)\end{array}$ & $\begin{array}{l}8(73 \%) \\
1(9 \%) \\
2(18 \%)\end{array}$ & $0.413^{\mathrm{ns}}$ & Chi square \\
\hline $\begin{array}{ll}\text { Coexisting medical conditions: } \\
\text { - } & \text { Steroid taking } \\
\text { - } & \text { Diabetes } \\
\text { - } & \text { Chemotherapy } \\
\text { - } & \text { Obesity }\end{array}$ & $\begin{array}{c}\text { may have more } \\
\text { than } 1 \\
2(9.5 \%) \\
7(33.3 \%) \\
8(38.1 \%) \\
4(19 \%) \\
21(100 \%)\end{array}$ & $\begin{array}{c}\text { may have more } \\
\text { than } 1 \\
1(5.1 \%) \\
10(52.7 \%) \\
5(26.3 \%) \\
3(15.9 \%) \\
19(100 \%)\end{array}$ & $\begin{array}{c}\text { may have more } \\
\text { than } 1 \\
1(5.1 \%) \\
10(52.7 \%) \\
5(26.3 \%) \\
3(15.9 \%) \\
19(100 \%)\end{array}$ & $0.9^{\text {ns }}$ & Chi square \\
\hline $\begin{array}{l}\text { Site } \\
\text { Maxilla } \\
\text { Mandible } \\
\text { Both }\end{array}$ & $\begin{array}{c}4(33.3 \%) \\
8(66.7 \%) \\
0\end{array}$ & $\begin{array}{c}2(16.7 \%) \\
10(84 \%) \\
1(8 \%)\end{array}$ & $\begin{array}{c}1(9 \%) \\
9(82 \%) \\
1(9 \%)\end{array}$ & $0.21^{\mathrm{ns}}$ & Chi square \\
\hline
\end{tabular}

ns= non-significant, $*$ significant

TABLE (2) Treatment results and significance of the difference using chi- square test

\begin{tabular}{|l|c|c|c|}
\hline & A & B & $8(73 \%)$ \\
\hline Complete resolution & $4(33.3 \%)$ & $6(50 \%)$ & $2(18 \%)$ \\
\hline Delayed resolution & $5(41.7 \%)$ & $4(33.3 \%)$ & $1(9 \%)$ \\
\hline No resolution & $3(25 \%)$ & 3.81 & $0.42^{\mathrm{ns}}$ \\
\hline $\mathrm{X}^{2}$ & & \multicolumn{2}{|c|}{} \\
\hline $\mathrm{P}$ & \multicolumn{2}{|c|}{} \\
\hline
\end{tabular}




\section{DISCUSSION}

Despite the fact that MRONJ has been reported for nearly a decade, the pathophysiologic mechanisms of the disease remain largely unknown, consequently, the treatment of MRONJ is still considered a challenge for health professionals, especially for dentists. The therapeutic modalities for MRONNJ varies from conservative modalities as prescription of antibiotics and oral antibacterial mouthwashes to surgical therapy necessary to create conditions that allow the complete resolution of symptoms ${ }^{(23)}$. The present study aims to find an effective surgical treatment protocol that exhibit high rate of success with low complication rate for treatment of MRONJ by comparing the healing outcome of the combined use of BMP-2 and L-PRF with the single use of either L-PRF alone or BMP2 alone, also we tried to analyze the association between MRONJ resolution and patient-specific factors that may influence the treatment outcome of MRONJ.

Platelet concentrates for surgical use are usually used for their local release of platelet growth factor to stimulate tissue healing and regeneration. Four main types of Platelet concentrates products can be easily defined, depending on their leukocyte content and fibrin architecture: pure platelet-rich plasma (PRP), pure platelet-rich fibrin (PRF), leukocyte and platelet-rich plasma, and leukocyte- and plateletrich fibrin. The four sorts vary in fibrin architecture; a factor that significantly influences the healing capability and the therapeutic protocol associated with each platelet concentrate type technology ${ }^{(24)}$. In the present study L-PRF, was chosen for its clinical characteristics that allow good adaptation in the unhealed defect found due to MRONJ ( PRF does not require heterogeneous agents and can be used as a graft material or barrier membrane). Pripatnanont et $\mathrm{al}^{(25)}$ found that PRF is composed of densely thick fibrin networks with activated platelets meshed among the fibrins, these dense fibrins provide a natural scaffold for storage and attachment of tissues and the stimulation of angiogenesis. In addition to the matrix scaffold, platelets provide and sustain the release of many growth factors in the wound area, in addition, leukocyte found inside L-PRF acts as an anti-infectious agent and has a role in immune regulation ${ }^{(26)}$.

Some previous studies demonstrated that a combination of necrotic bone curettage with PRF application is a promising treatment for MRONJ(27-28), PRF can enhance wound healing and bone maturation. However, there is not sufficient studies on whether it promotes soft tissue healing in MRONJ cases or not. A review reported the slow release of key growth factors by PRF, with the growth factors released for at least 1 week and up to 28 days $^{(29)}$. Accordingly, PRF could stimulate the release of growth factors for a long time during wound healing.

MRONJ is understood as a disease mainly associated with the oversuppression of bone remodeling as the main pharmacological effect of antiresorptive drugs is inhibition of osteoclast. BMP2 not only can stimulate osteoblast differentiation and proliferation owing to its osteoinductive capacity which has been demonstrated in numerous studies $^{(18)}$, but also BMP-2 has a biphasic function of bone resorption by directly stimulating osteoclast activation and differentiation, which is viewed as an administrative instrument of bone mass as reported by several in vitro studies ${ }^{(30-31-32)}$. In an animal study, insertion of BMP-2 into the tooth sockets of dogs that was previously exposed to zoledronic acid was demonstrated to counteract the negative effects of zoledronic acid on bone healing and remodeling ${ }^{(21)}$. In addition, a clinical trial also supported the feasibility of BMP-2 in the treatment of $\mathrm{MRONJ}^{(33)}$. Through biphasic function, BMP-2 seems to have an inversion impact on remodeling suppressed bone of MRONJ, so enhance bone remodeling.

The present study showed significant association between resolution of MRONJ and the combined therapy of L-PRF and BMP-2 compared to the single therapy of L-PRF or BMP-2, this was clear 
in the variation of the healing periods, but the difference was not significant which may be due to the small sample size we worked upon. Patients in the PRF group and the BMP-2 groups showed more delayed healing patterns than that of the PRF plus BMP group, also the BMP-2 group showed superior result compared to the L-PRF group. Consequently, according to the AAOMS guideline that suggested to prioritize continued antiresorptive therapy ${ }^{(3)}$, patients may benefit from this combined therapy since early healing of lesion can allow continuous antiresorptive therapy, BMP-2 enmeshed in the L-PRF matrix has angiotrophic, haemostatic, and osseous conductive properties ${ }^{(34)}$. So collectively, In group $\mathrm{C}$ where enmeshed BMP found in fibrin matrix such as L-PRF had stimulated both soft tissue healing and also bone remodeling, this might contribute in giving the best outcomes and effective treatment of MRONJ .

On the other hand, patients treated with the single use of PRF or BMP-2 showed more delayed healing patterns than that treated with both of them at the same time this may be explained by the absence of BMPs within L-PRF in group A, making the condition doubtful whether it is an effective treatment for MRONJ because MRONJ has been primarily thought to be an osseous disease. Also the healing outcome for the group B was inferior which may be explained due to the absence of L-PRF matrix scaffold that supports the BMP-2 and the absence of the slow release of key growth factors mechanism found in the L-PRF.

Similar to our protocol and despite the opinions that conservative non surgical management is the preferred method of MRONJ treatment, there appears to be a new trend of surgical treatment which shows predictable healing outcomes with a success rate of $73-100 \%{ }^{(34)}$, predictable with this, a systematic review reported that the average healing rates for surgical management of MRONJ were $84 \%$ for extensive surgery, $85 \%$ for extensive laser-assisted surgery and $75 \%$ for conservative surgery ${ }^{(35)}$. However, the results of nonsurgical management were lower than the surgical numbers with healing rates of $36 \%$ for antibiotic treatment alone, $30-52 \%$ for antibiotic therapy in combination with hyperbaric oxygen therapy or low level laser therapy ${ }^{(36)}$.

\section{IN CONCLUSION}

The present study demonstrated a significant association between the combined regimen of L-PRF and BMP-2 and the resolution of MRONJ with the least complication rates compared to the single use of L-PRF or BMP-2. Neither PRF nor rhBMP-2 have been used on wide scale in the treatment of MRONJ. Therefore further investigations on a greater population number are needed to emphasize whether this combination provides added benefits over the standard of care or not.

\section{REFERENCES}

1- Russell RG: Bisphosphonates: the first 40 years. Bone $49: 2,2011$.

2- Wu S, Dahut WL, Gulley JL: The use of bisphosphonates in cancer patients. Acta Oncol 46:581, 2007.

3- Ruggiero SL, Dodson TB, Fantasia J, et al: American Association of Oral and Maxillofacial Surgeons Position Paper on Medication-Related Osteonecrosis of the Jaw2014 Update. Journal of Oral and Maxillofacial Surgery $72: 1938,2014$.

4- Marx RE: Pamidronate (Aredia) and zoledronate (Zometa) induced avascular necrosis of the jaws: A growing epidemic. J Oral Maxillofacial Surg 61:1115, 2003.

5- American Association of Oral and Maxillofacial Surgeons: Position paper on bisphosphonate-related osteonecrosis of the jaws: Advisory Task Force on Bisphosphonate-Related Osteonecrosis of the Jaws, American Association of Oral and Maxillofacial Surgeons. J Oral Maxillofac Surg 65:369, 2007.

6- Ruggiero SL, Mehrotra B, Rosenberg TJ, et al: Osteonecrosis of the jaws associated with the use of bisphosphonates: A review of 63 cases. J Oral Maxillofac Surg 62:527, 2004.

7- Migliorati CA: Bisphosphonates and oral cavity avascular bone necrosis. J Clin 21:4253, 2003. 
8- Ruggiero, Salvatore L, et al. "American Association of Oral and Maxillofacial Surgeons position paper on medication-related osteonecrosis of the jaw-2014 update." Journal of Oral and Maxillofacial Surgery 72.10: 19381956, 2014.

9- Advisory Task Force on Bisphosphonate-Related Ostenonecrosis of the Jaws AAoO, Maxillofacial S: American Association of Oral and Maxillofacial Surgeons position paper on bisphosphonate-related osteonecrosis of the jaws. J Oral Maxillofac Surg 65:369, 2007.

10- Holzinger D, Seemann R, Klug C, Ewers R, Millesi G, Baumann A, Wutzl A: Long-term success of surgery in bisphosphonate-related osteonecrosis of the jaws (BRONJs). Oral Oncol 49:66, 2013.

11- Ruggiero SL, Kohn N: Disease Stage and Mode of Therapy Are Important Determinants of Treatment Outcomes for Medication-Related Osteonecrosis of the Jaw. J Oral Maxillofac Surg 73:S94, 2015.

12- Wutzl A, Pohl S, Sulzbacher I, Seemann R, Lauer G, Ewers R, Drach J, Klug C: Factors influencing surgical treatment of bisphosphonate-related osteonecrosis of the jaws. Head Neck 34:194, 2012.

13- Lopes RN, Rabelo GD, Rocha AC, Carvalho PA, Alves FA: Surgical Therapy for Bisphosphonate- Related Osteonecrosis of the Jaw: Six-Year Experience of a Single Institution. J Oral Maxillofac Surg 73:1288, 2015.

14- Choukroun J, Adda F, Schoeffer C, et al: PRF: An opportunity in perio-implantology. Implantodontie 42:55, 2000.

15- Dhoan DM, Choukroun J, Diss A, et al: Platelet-rich fibrin (PRF) :A second-generation platelet concentrate. Part II: Platelet related biologic features. Oral Surg Oral Med Oral Pathol Oral Radiol Endod 101:45, 2006.

16- American Association of Oral and Maxillofacial Surgeons J Oral Maxillofac Surg -:1-5, 2013. PRF may aid wound healing and the closure of bone in MRONJ.

17- Urist MR, Strates BS: Bone morphogenetic protein. J Dent Res 50:1392, 1971

18- Chen D, Zhao M, Mundy GR: Bone morphogenetic proteins. Growth Factors 22:233, 2004.

19- Jones AA, Buser D, Schenk R, et al: The effect of rhBMP2 around endosseous implants with and without membranes in a canine model. J Periodontol 77:1184, 2006.

20- Visser R, Arrabal PM, Becerra J, et al: The effect of an rhBMP2 absorbable collagen sponge-targeted system on bone formation in vivo. Biomaterials 30:2032, 2009.
21- Gerard DA, Carlson ER, Gotcher JE, et al: Early inhibitory effects of zoledronic acid in tooth extraction sockets in dogs are negated by recombinant human bone morphogenetic protein. Journal of Oral and Maxillofacial Surgery 72:61, 2014.

22- Reid IR, Cornish J: Epidemiology and pathogenesis of osteonecrosis of the jaw. Nature Reviews Rheumatology 8:90, 201.

23- Ruggiero SL, Dodson TB, Assael LA, et al: American Association of Oral and Maxillofacial Surgeons position paper on bisphosphonate-related osteonecrosis of the jaws 2009 Update. J Oral Maxillofac Surg 67:2, 2009.

24- Dohan Ehrenfest DM, Bielecki T, Jimbo R, et al: Do the fibrin architecture and leukocyte content influence the growth factor release of platelet concentrates? An evidence-based answer comparing a pure platelet-rich plasma (P-PRP) gel and a leukocyte- and platelet-rich fibrin (LPRF). Curr Pharm Biotechnol 13:1145, 2012.

25- Mawardi H, Giro G, Kajiya M, et al: A role of oral bacteria in bisphosphonate-induced osteonecrosis of the jaw. J Dent Res 90:1339, 2011.

26- Migliorati CA, Siegel MA, Elting LS: Bisphosphonateassociated osteonecrosis: A long term complication of bisphosphonate treatment. Lancet Oncol 7:508, 2006.

27- Anitua E, Troya M, Orive G: Plasma rich in growth factors promotes gingival tissue regeneration by stimulating fibroblast proliferation and migration and by blocking TGF-b1induced myodifferentiation. J Periodontol 83:1028, 2012.

28- Mozzati M, Gallesio G, Arata V, et al: Platelet-rich therapies in the treatment of intravenous bisphosphonate-related osteonecrosis of the jaw: A report of 32 cases. Oral Oncol 48:469, 2012 19. Anitua E, Troya M, Orive G: Plasma rich in growth factors promotes gingival tissue regeneration by stimulating fibroblast proliferation and migration and by blocking TGF-b1-induced myodifferentiation. J Periodontol 83:1028, 2012.

29- Ferlito S, Puzzo S, Liardo C: Preventive protocol for tooth extractions in patients treated with zoledronate: A case series. J Oral Maxillofac Surg 69:e1, 2011.

30- Kim RY, Oh JH, Lee BS, et al: The effect of dose on rhBMP-2 signaling, delivered via collagen sponge, on osteoclast activation and in vivo bone resorption. Biomaterials 35:1869, 2014. 
31- Kaneko H, Arakawa T, Mano H, et al: Direct stimulation of osteoclastic bone resorption by bone morphogenetic protein (BMP)-2 and expression of BMP receptors in mature osteoclasts. Bone 27:479,2000.

32- Itoh K, Udagawa N, Katagiri T, et al: Bone morphogenetic protein 2 stimulates osteoclast differentiation and survival supported by receptor activator of nuclear factor- $\varkappa \mathrm{B}$ ligand. Endocrinology 142:3656, 2001.

33- Cicciù M, Herford AS, Juodžbalys G, et al: Recombinant human bone morphogenetic protein type 2 application for a possible treatment of bisphosphonates-related osteonecrosis of the jaw. Journal of Craniofacial Surgery 23:784, 2012.
34- Kim J-W, Kim S-J, Kim M-R: Bone morphogenetic protein-2 and Platelet-rich Fibrin for treatment of bisphosphonate-related osteonecrosis of jaw. Journal of Oral Implantology, 2015.

35- Carlson ER: Management of antiresorptive osteonecrosis of the jaws with primary surgical resection. J Oral Maxillofac Surg 72:655, 2014.

36- Rupel K, Ottaviani G, Gobbo M, et al: A systematic review of therapeutical approaches in bisphosphonates-relate osteonecrosis of the jaw (BRONJ). Oral oncology 50:1049, 2014. 